Scientific Paper

\title{
A Hybrid Fuzzy-SVM classifier for automated lung diseases diagnosis
}

\author{
Donia Ben HASSEN ${ }^{1, a}$, Sihem Ben ZAKOUR ${ }^{1}$, Hassen TALEB ${ }^{2}$ \\ ${ }^{1}$ Faculty of law, economics and management of Jendouba, University of Jendouba, Tunisia \\ ${ }^{2}$ Faculty of economics and management of Nebeul, University of Carthage, Tunisia \\ ${ }^{a}$ E-mail address: donia_ben_hassen@yahoo.fr
}

(received 30 June 2016; revised 27 November 2016 and 1 December 2016; accepted 13 December 2016)

\begin{abstract}
A novel scheme for lesions classification in chest radiographs is presented in this paper. Features are extracted from detected lesions from lung regions which are segmented automatically. Then, we needed to eliminate redundant variables from the subset extracted because they affect the performance of the classification. We used Stepwise Forward Selection and Principal Components Analysis. Then, we obtained two subsets of features. We finally experimented the Stepwise/FCM/SVM classification and the PCA/FCM/SVM one. The ROC curves show that the hybrid $\mathrm{PCA} / \mathrm{FCM} / \mathrm{SVM}$ has relatively better accuracy and remarkable higher efficiency. Experimental results suggest that this approach may be helpful to radiologists for reading chest images.
\end{abstract}

Key words: computer aided diagnosis; lung lesion classification; FCM; SVM; PCA.

\section{Introduction}

By 2020, four of the seven major killers worldwide are expected to be lung diseases [1]. A chest X-ray is often the first procedure a patient will undergo if the doctor suspects he has lung disease. Uncertainty is widely present in data in chest radiography. Computer-assisted approaches may be helpful for handling this vagueness and as a support to diagnosis in this field. Therefore, the development of a reliable computer aided diagnosis (CAD) system for lung diseases is one of the most important research topics. Despite, lesion classification systems provide the foundation for lesions diagnosis and patient cure, the studies reported in developing a CAD application was limited to the distinction between the cancerous lesions from the non-cancerous. Physicians need a system which is significantly analogous to a human judgment in the process of analysis and decision making. The design of a classifier which can give an idea about the nature of the lesion, for example the lesion is of 50\% an infection, $10 \%$ a cancer and $30 \%$ a tuberculosis etc... can help the radiologist to be suitable for handling a decision making process concerning. To reach this goal, we propose a novel approach for classification of chest lesions.

The remaining parts of this paper are arranged as follows: after considering related works in section 2, section 3 describes the computerized scheme for classification of lung lesions. Then, the proposed methods are detailed in section 4. Corresponding experiments are shown in the Experimental Results part; the following part gives the conclusions of this study.

\section{Related Works}

The clinical importance of chest radiographs and their complicated nature induces to develop many Computer Aided Diagnostic systems (CAD) to assist doctors in the detection and classification of lung lesions.

The specification of the nature of lesions on chest radiographs is an important task also for radiologists. But they may fail to classify them. The wrong diagnostic can guide the analysis to the incorrect path and cause the loss of time and extra expenses. That's why the development of a reliable computer aided diagnosis (CAD) system for lung disease is one of the most important research topics. There are two major categories related to the CAD systems: CADe (Computer Aided Detection) and CADx (Computer Aided Diagnosis) [2]. CADe systems detect lesions through medical images (CADe systems are outside the scope of this work). While CADx systems aim to improve the processing speed and determining the malignancy of the lesion. We are interested in our work to this category which is differentiating between malignant and benign lesions.

More importantly, most research efforts in CADx have concentrated on classification of nodules to benign and malign. Experiments in which lesions are categorized as benign or malignant together with clinical findings are automatically diagnosed, have shown very promising results. But we think that categorizing lesion in which lung disease belongs to, may become an important aid to the radiologist in diagnosis.

Many methods have been proposed in the literature for chest lesions classification and diagnosis utilizing a wide variety of algorithms. The majority of researches include the 
classification process under a description of whole Computer Aided Diagnosis systems [2].

The first scientific article that used the term computer-aided diagnosis (CAD), written by Lodwick in 1966. In 1966, [3] published a study on the analysis of pulmonary nodules detected in chest radiographs. He used features that were visually determined by human experts from chest radiographs as input for a Bayesian classifier, and the classifier determined the probability that the nodules were cancerous. The first application of CAD was thus in chest radiology. Following the vision of Lodwick, CAD in chest radiography was actively researched in the 1970s [1]. Within this context of work, a system which automatically detects and classifies lung lesions from chest radiographs was proposed in [4]. The system extracts a set of candidate regions by applying to the radiograph three different multi-scale schemes. Support Vector Machines (SVMs), using as input different sets of features, has been successfully applied for the classification of chest lesions to benign and malign. The work of [5] has considered the classification process as distinction between true lesions and normal tissues in order to improve radiologists' accuracy in detecting lung nodules.

Fuzzy Logic based methods [6-7] are often used to finish data classification tasks and usually can get good classification results. The authors in [8] have developed and evaluated a Computer-Aided Diagnostic algorithm for lung nodule characterization and classification in Chest Radiographs using Multi-scale Wavelet.

An optimized Support Vector Machine classifier was proposed in [9]. The main goals of this study are to improve the classification efficiency and accuracy of SVM in the medical image.

The work of [10] proposes a methodology to classify lung nodule and non-nodule using texture features, artificial crawlers and the rose diagram.

We found that the majority of works in Computer Aided Diagnosis systems in chest radiography has focused on lung cancerous nodule detection. Considering the load of lung diseases and the position of chest radiography in the diagnostic workflow of these diseases, we could argue that the classification of other types of lesions should receive more attention. We also think that an adapted framework for extraction of the adopted features describing the different kinds of lesions is the missing part of the methods presented in the literature.

\section{Computerized Scheme for Classification of Lung Lesions}

Here, we only describe the final stage of our CAD system which is a continuation of our already published work in [11]: The segmentation and the detection are discussed in our previous works [12] and [13] that are briefly presented in the next sections.

\subsection{Segmentation}

We closely follow our previous work described in [12] which is an automatic chest radiography segmentation framework with integration of spatial relations. The algorithm developed as the initial step of our system works under no assumption. The results obtained proving that this is an excellent initialization step for a CAD system aimed at lung lesions detection and recognition of their sites. The segmented lung area includes even those parts of the lungs which are usually excluded from the methods presented in the literature.

\subsection{Feature extraction}

Almost all features presented in the literature are classical features. Some of them describe the shape of the candidate region considered as the circularity, the perimeter; some features are used to study the texture such as the intensity. In this stage, we need new features that should be able to represent the characteristics of the lesions and specify the nature of the detected lesion.

To this aim, we computed several features based on characteristics given by service of medical imaging of $\mathrm{CHU}$ Charles Nicolle (described in our published work [11]).

We discussed with our collaborators in the service of medical imaging of the university hospital Charles Nicolle and we detected the five important diseases which are: Lung cancer, Metastasis, Tuberculosis, Infection, Benign tumors and we computed the correspond features:

- The $\boldsymbol{X}$-fraction and $\boldsymbol{Y}$-fraction: there are the coordinates of the centroid of the lesion.

- The circularity is defined as the fraction of the area of the region contained in the circle, with the same area and centered in the center of mass, and the area of the circle itself.

- Skewness which measures the degree of asymmetry values of the color components relative to their average.

- Kurtosis that estimates the degree of concavity or convexity of the color components relative to their average. A positive kurtosis indicates a relatively sharp distribution, while a negative kurtosis indicates a relatively flat distribution.

- Entropy provides information about the disorder that can have the texture.

- Correlation measures the linear dependencies between the levels of the components color in the image.

- Homogeneity reflects local homogeneity in the texture.

- Lacunarity describes the distribution of gaps within a texture.

- Localisation: This feature is calculated thanks to the information obtained with the segmentation. It takes a value equal to 1 if the centroid is in right apical, 2 if the centroid is in right axillary, 3 if the centroid is in right paracardiac, 4 if the centroid is in right basal, 5 if the centroid is in left apical, 6 if the centroid is in left axillary, 7 if the centroid is in left paracardiac, 8 if the centroid is in left basal. 
In clinic practice, there are other descriptors highly discriminant. These information is generally recorded in the patient file or attached to the image in DICOM format. These descriptors can provide several useful insights in the description of the image content. For example, a smoker patient is more likely to have lung cancer than non-smokers individual. Our collaborators in CHU Charles Nicolle have proposed this clinical description detailed in Table $\mathbf{1}$ below.

Table 1. Clinical attributes according to radiologists

\begin{tabular}{lcccc}
\hline \hline Metestasis & $\begin{array}{c}\text { Benign } \\
\text { tumors }\end{array}$ & Malign tumors & Tuberculosis \\
\hline Age & aged & young & aged & young \\
\hline Gender & indifferent & indifferent & $\begin{array}{c}\text { men are exhibited } \\
\text { more than women }\end{array}$ & indifferent \\
\hline Smoking & & & $\begin{array}{c}\text { smoking is very } \\
\text { related to this disease }\end{array}$ & \\
\hline \hline
\end{tabular}

The JSRT database, which is a publicly available database with 247 Posterior-Anterior chest radiographs [14], contains only these two descriptors: the age and the gender. In the total we have twelve descriptors.

\section{The proposed methods}

\subsection{Feature Reduction}

To be classified, images are shown as projected in a space image points called feature space. Feature reduction is a very important step in organizing a classifier. It eliminates insignificant and correlated features that may deteriorate the quality of the discrimination. Reduction methods can be divided into two categories: features selection and features extraction. The feature selection is to choose from among the calculated attributes, a small number of them that are most relevant.

However, feature extraction is to extract from the initial set of attributes, a new smaller set, which contains the same information [15].

The number of reduction methods is very important; the Figure 1 below presents a brief overview of the main methods.

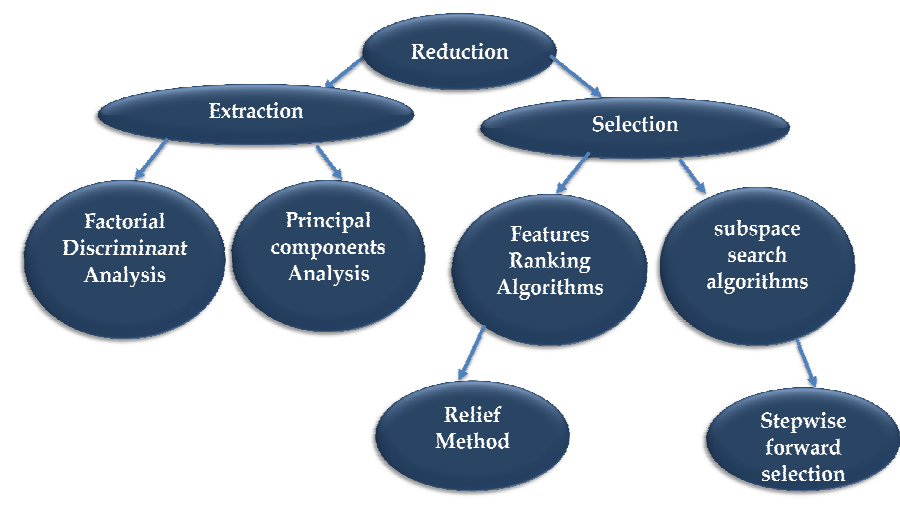

Figure 1. Overview of the main methods of feature selection

\subsection{The process of Principal Component Analysis}

Principal Component Analysis (PCA) is defined as a classical dimension reduction method for feature selection and data representation technique widely used in the areas of Computer Aided Diagnosis System [16]. Eigenvalue and eigenvector components are ranked according to their variance to the principal axes and ranked from having the most contribution to the least one. Consider the following.

Let $x$ be an n-dimensional column vector. The project image $A$ is an $m n_{x}$ matrix, onto $x$ by $y=A_{x}$. In order to determine the optimal projection vector $x$, the total scatter of the projected samples $S_{x}$ is utilized to measure the optimality of $x$ :

$\mathrm{S}_{\mathrm{x}}=\mathrm{x}^{\mathrm{t}} \mathrm{E}\left\{[\mathrm{A}-\mathrm{E}(\mathrm{A})]^{\mathrm{T}}[\mathrm{A}-\mathrm{E}(\mathrm{A})]\right\} \mathrm{x}=\mathrm{x}^{\mathrm{T}} \mathrm{S}_{\mathrm{A}} \mathrm{x}$

Eq. 1

where $\mathrm{S}_{\mathrm{A}}$ depicts the image covariance matrix. Suppose that there are $\mathrm{M}$ training samples $\mathrm{A}_{\mathrm{i}}\{\mathrm{i}=1,2,3, \ldots, \mathrm{M}\}$ and $\bar{A}$ is the average image:

$\mathrm{S}_{\mathrm{A}}=\frac{1}{\mathrm{M}} \sum_{\mathrm{i}=1}^{\mathrm{M}}\left[\mathrm{A}_{\mathrm{i}}-\overline{\mathrm{A}}\right]^{\mathrm{T}}\left[\mathrm{A}_{\mathrm{i}}-\overline{\mathrm{A}}\right]$

Eq. 2

The optimal projection direction $\mathrm{x}_{\mathrm{opt}}$ denotes the eigenvector of $\mathrm{S}_{\mathrm{A}}$ corresponding to the largest eigenvalue. Usually a set of orthonormal projection directions, $x_{1}, x_{2}, \ldots x_{d}$, are chosen. These projection directions are the orthonormal eigenvectors of $\mathrm{S}_{\mathrm{A}}$ corresponding to the first $\mathrm{d}$ largest eigenvalues.

For a given A, let $y_{k}=A x_{k}\{k=1,2, \ldots, d\}$. A set of projected feature vector $y_{k}$ and the principal components of $\mathrm{A}$ are found. The feature matrix of $\mathrm{A}$ is obtained as $B=\left[y_{1}, y_{2}, \ldots, y_{d}\right]$. The nearest neighborhood classifier is adopted for classification. The distance between two arbitrary feature matrices, $B_{i}$ and $B_{j}$, is given by:

$s d\left(\left(B_{i}, B_{j}\right)=\sum_{k=1}^{d}\left\|y_{k}^{i}-y_{k}^{j}\right\|_{2}\right.$

Eq. 3

Where $\left\|y_{k}^{i}-y_{k}^{j}\right\|_{2}$ depicts the Euclidean distance between $\mathrm{y}_{\mathrm{k}}^{\mathrm{i}}$ and $\mathrm{y}_{\mathrm{k}}^{\mathrm{j}}[16]$.

\subsection{Selection of the most discriminant Features}

The reduction in the feature space in the context of the classification of medical radiographic images is a primordial step. First, it eliminates insignificant or irrelevant features. On the other hand, high number of features may deteriorate the quality of the classification in addition to the risk of correlated features. Finally, the time required for the classification depends on how many features are considered. The main methods used to reduce the dimension of the feature space have been presented in the previous section. We saw that it is better to use search algorithms subspace rather than ranking algorithms attributes. Indeed, they measure the relevance of attributes with appreciation individually; they do not eliminate the redundant attributes. Therefore, we look to search subspace algorithms especially the stepwise forward selection.

It thus seems interesting to compare the relevance of attributes resulting from a selection method with those from an extraction method. We propose to realize the classification 
phase with two sets of features in order to know the most pertinent features.

We propose to compare the results of the use of the stepwise forward selection with the results of an extraction method which is Principal Components Analysis (PCA).

The motive force behind the use Principal Component Analysis is to avoid the selection of redundant variables. It doesn't need any prototypes unlike Factorial Discriminant Analysis (FDA). However, its limit is that it is a projection method, and that the loss of information caused by the projection may cause misinterpretation. However there are tips to avoid these disadvantages which will be used in the experimentation part.

\subsection{Experimental results}

This section describes the details of the experiments that we performed in order to show the results of classification obtained by applying the Stepwise Forward Selection and the Principal Components Analysis to the calculated features. We start by providing a description of the used data sets. Then, we explain the results of the selection of significant features. After that, Different experimentations concerning the classification using FCM, SVM and FCM-SVM are presented. Finally, we give the discussion.

The forward stepwise selection method was adopted to find the optimal combination of features among 12 features. The leave-one-out method was employed to evaluate the performance of each combination of features. The results gave 8 features (size, circularity, $\mathrm{x}$-fraction, $\mathrm{y}$-fraction, skewness, kurtosis, homogeneity, correlation).

The graph (Figure 2) shows circle of correlation. It corresponds to a projection of the variables on a twodimensional plane formed by the two factors. When two variables are far from the center of the graph, while if they are: the close relative to each other, while they are significantly positively correlated, orthogonal with respect to each other, while they are significantly uncorrelated symmetrically opposed relative at the center, then they are negatively correlated significantly.

The first result is interesting to analyze the correlation matrix (Table 2). We note the result that the Size is correlated respectively with $\mathbf{X}$-fraction and $\mathbf{Y}$-fraction $(\mathrm{r}=0.924)$ $(\mathrm{r}=0.922)$. We could notice also the skewness is perfectly correlated with the kurtosis $(r=-1)$, they are redundant variables. The same thing for the $\mathbf{X}$-fraction and the $\mathbf{Y}$-fraction $(r=0.999)$.

By referring to these factorial planes and taking into account of comments provided above, we can infer that the $\mathbf{X}$-fraction, the Y-fraction and the Skewness variables should be suppressed and we can deduce that only ten variables are not correlated.

In the next section, we will use these two subsets of features in many experiments to conclude the optimal subset and the good classifier.

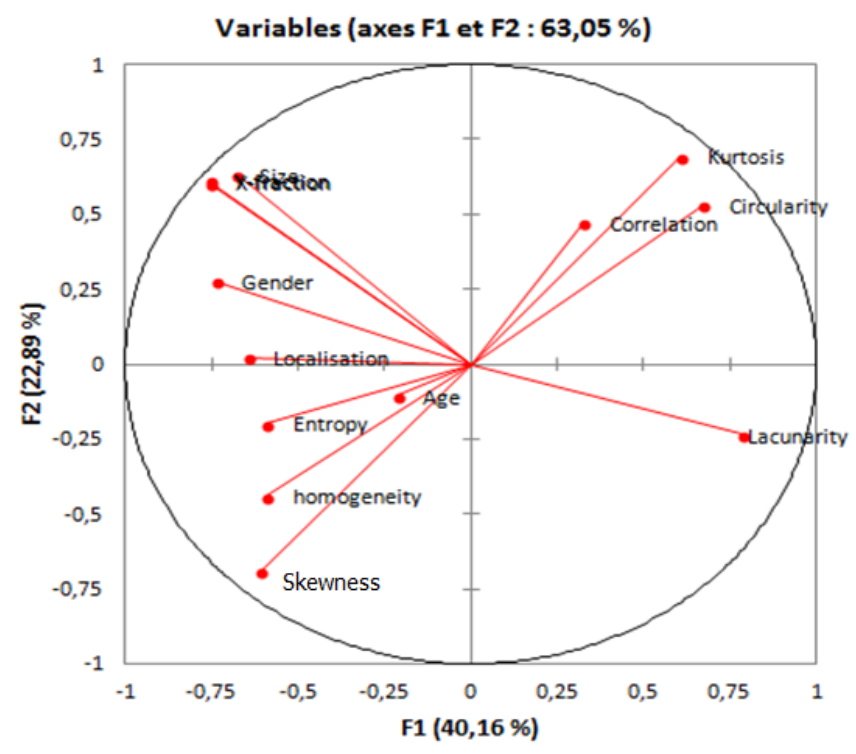

Figure 2. Circle of correlation

Table 2. Matrix of Pearson

\begin{tabular}{|c|c|c|c|c|c|c|c|c|c|c|c|c|c|}
\hline Variables & $\stackrel{\tilde{N}}{\tilde{n}}$ & $\underset{\&}{\infty}$ & ن & $\frac{\dot{0}}{\bar{D}}$ & 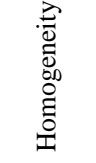 & 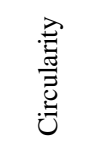 & \begin{tabular}{l}
$\tilde{d}$ \\
\multirow{2}{0}{} \\
$\frac{\tilde{v}}{v}$
\end{tabular} & $\begin{array}{l}\frac{n}{5} \\
\stackrel{0}{\Xi} \\
\underline{\Xi}\end{array}$ & $\begin{array}{l}\stackrel{\Xi}{\Xi} \\
\stackrel{\Xi}{\Xi} \\
\stackrel{\Xi}{\Xi}\end{array}$ & 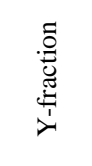 & 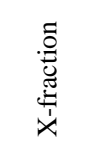 & $\underset{\text { 艺 }}{\stackrel{Z}{0}}$ & 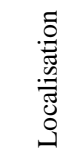 \\
\hline Size & 1 & -0.032 & 0.129 & 0.582 & 0.192 & -0.119 & 0.059 & -0.059 & -0.689 & 0.922 & 0.924 & 0.178 & 0.281 \\
\hline Age & -0.032 & 1 & 0.020 & 0.206 & -0.060 & -0.232 & 0.095 & -0.095 & -0.149 & -0.008 & -0.023 & 0.266 & 0.453 \\
\hline Correlation & 0.129 & 0.020 & 1 & -0.133 & -0.308 & 0.422 & -0.377 & 0.377 & 0.382 & 0.052 & 0.053 & -0.176 & -0.191 \\
\hline Gender & 0.582 & 0.206 & -0.133 & 1 & 0.295 & -0.412 & 0.204 & -0.204 & -0.474 & 0.693 & 0.697 & 0.358 & 0.445 \\
\hline Homogeneity & 0.192 & -0.060 & -0.308 & 0.295 & 1 & -0.506 & 0.640 & -0.640 & -0.320 & 0.177 & 0.179 & 0.399 & 0.288 \\
\hline Circularity & -0.119 & -0.232 & 0.422 & -0.412 & -0.506 & 1 & -0.779 & 0.779 & 0.298 & -0.230 & -0.237 & -0.358 & -0.322 \\
\hline Skewness & 0.059 & 0.095 & -0.377 & 0.204 & 0.640 & -0.779 & 1 & -1.000 & -0.284 & 0.088 & 0.101 & 0.373 & 0.211 \\
\hline Kurtosis & -0.059 & -0.095 & 0.377 & -0.204 & -0.640 & 0.779 & -1.000 & 1 & 0.284 & -0.088 & -0.101 & -0.373 & -0.211 \\
\hline Lacunarity & -0.689 & -0.149 & 0.382 & -0.474 & -0.320 & 0.298 & -0.284 & 0.284 & 1 & -0.700 & -0.694 & -0.355 & -0.541 \\
\hline Y-fraction & 0.922 & -0.008 & 0.052 & 0.693 & 0.177 & -0.230 & 0.088 & -0.088 & -0.700 & 1 & 0.999 & 0.239 & 0.404 \\
\hline$X$-fraction & 0.924 & -0.023 & 0.053 & 0.697 & 0.179 & -0.237 & 0.101 & -0.101 & -0.694 & 0.999 & 1 & 0.240 & 0.376 \\
\hline Entropy & 0.178 & 0.266 & -0.176 & 0.358 & 0.399 & -0.358 & 0.373 & -0.373 & -0.355 & 0.239 & 0.240 & 1 & 0.661 \\
\hline Localisation & 0.281 & 0.453 & -0.191 & 0.445 & 0.288 & -0.322 & 0.211 & -0.211 & -0.541 & 0.404 & 0.376 & 0.661 & 1 \\
\hline
\end{tabular}




\section{Description of the Classification System}

The classification problem has been addressed in many computing applications such as medical diagnosis. In the literature we can find the term grouping or clustering instead of classification. Although these two terms are similar in terms of language, they are technically different. In fact, classification is a discriminant analysis that uses a supervised approach where we are provided with a collection of labeled data; the problem is to label a newly unlabeled data. Usually, the given training data is used to learn the descriptions of classes which in turn are used to label a new data. Among these methods are Bayes classifier, artificial neural networks, deformable models and support vector machines. Normally, clustering always refers to unsupervised framework which its problem is to group a given collection of unlabeled patterns into meaningful clusters. There is a whole family of unsupervised methods, including probabilistic ones, fuzzy ones, evidential ones. Especially, there are two variants of unsupervised classifiers: classifiers are known as hard methods and fuzzy methods. The commonly used fuzzy clustering methods are: FCM Fuzzy C- Means and its variants. The detail of this part is already published in our previous work in [11].

Table 3. Performances of FCM and SVM classifier in our dataset [11]

\begin{tabular}{ccccccc}
\hline \hline \multirow{2}{*}{ Classes } & \multicolumn{2}{c}{ Acccuracy (\%) } & \multicolumn{2}{c}{ SE(\%) } & \multicolumn{2}{c}{ SP(\%) } \\
\cline { 2 - 7 } & FCM & SVM & FCM & SVM & FCM & SVM \\
\hline Cancer & 70.33 & 74.19 & 73.00 & 80.00 & 71.33 & 60.00 \\
\hline Metastasis & 60.25 & 50.94 & 63.88 & 66.66 & 61.56 & 50.00 \\
\hline Infection & 55.66 & 51.72 & 52.76 & 50.00 & 53.00 & 52.00 \\
\hline Tuberculosis & 60.23 & 56.56 & 55.85 & 53.33 & 57.00 & 51.88 \\
\hline Benign tumors & 53.33 & 52.00 & 54.67 & 51.45 & 53.89 & 50.00 \\
\hline \hline
\end{tabular}

\subsection{Methods and results}

We judge the performance of our classification approach using several evaluation criteria often used in the literature. For a five class clustering problem, one can distinguish true positive (TP) (sample correctly classified), false positive (FP) (false sample classified as true sample), false negative (FN) (false sample classifier as false sample), and true negative (TN) (false sample classified as true sample).

From these values, measures such as accuracy, sensitivity (SE) and specificity (SP) can be computed given by the following equations.

$S E=\frac{T P}{T P+F N}$
$S P=\frac{T N}{T N+F P}$

Eq. 4

Accuracy $=\frac{T P+T N}{T P+T N+F P+F N}$

The results in Table 3 show that the clusters are identified well by the FCM algorithm.
The clusters that contain more errors are those that contain fewer samples. The lung cancer is the cluster which is more clearly identified by the FCM algorithm (achieved an averaged accuracy rate of the order $70.33 \%$ for cancer).

The obtained results by SVM are satisfactory. Indeed, we reached a recognition rate of the order $74.19 \%$ for the cancer. We remark that the classification rate is less in the other classes because of the fewer number of the train and the test data.

We can conclude that the SVM can achieve better accuracy in our classification problem. However, the two methods cannot represent clusters of small size.

The Fuzzy C Means Algorithm uses a fuzzy clustering, in which the input vector $\mathrm{x}$ is pre-classified with different membership values. The outputs of the FCM algorithm may present the input vector to the SVM classifier. This last will be used for the automatic recognition of disease.

The intelligibility is the motive force behind the use of FCM algorithm for this problem. However, a compromise between interpretability and accuracy is met. On the other hand, we focused on a more accurate solution by using SVM. Then we risk losing the linguistic sense defining the fuzzy models. Indeed, we have experimented also the possibility to increase the interpretability of SVM classifier by the hybridization with the clustering method Fuzzy $\mathrm{C}$ means. The figures below show the results of Stepwise/FCM/SVM classification and PCA/FCM/SVM one.

A Receiver Operating Characteristic curve (or ROC curve) is a plot of the true positive rate against the false positive rate (sensitivity (y-axis) vs. 1 - specificity ( $\mathrm{x}$-axis)) for the different possible cut points of a diagnostic test.

Each point on the ROC curve represents a sensitivity/specificity pair corresponding to a particular decision threshold. The area under the ROC curve (AUC) is an indicator of the classification performance [18]. A value of $\mathrm{AUC}=1$ represents perfect classification, $\mathrm{AUC}=0.5$ corresponds to random guessing. AUC and its standard deviation are computed by fitting a bi-normal model to the ROC data.

The results obtained with the 8 features of stepwise forward selection are comparable to those obtained with the 10 features of the Principal Components Analysis. The first subset of features has too low a discriminating power for the five classes of diseases. It is interesting to note that the performance of $\mathrm{AUC}=0.884$ on the cancer database with PCA features is much higher than the AUC value of the Stepwise features, as shown in Figure 4, that do not exceed 0.699.

These results are better than those presented above in Table 3, proving that the hybridization between FCM and SVM greatly improve classifier performance.

SVM and FCM are promising algorithms, which have solid theory foundation and good generalization ability. But correlated features can extremely affect the quality of classification. In this paper, PCA is adopted to reduce the dimension of our data. As shown in our experiments, hybridizing PCA, FCM and SVM can assure classification accuracy. 

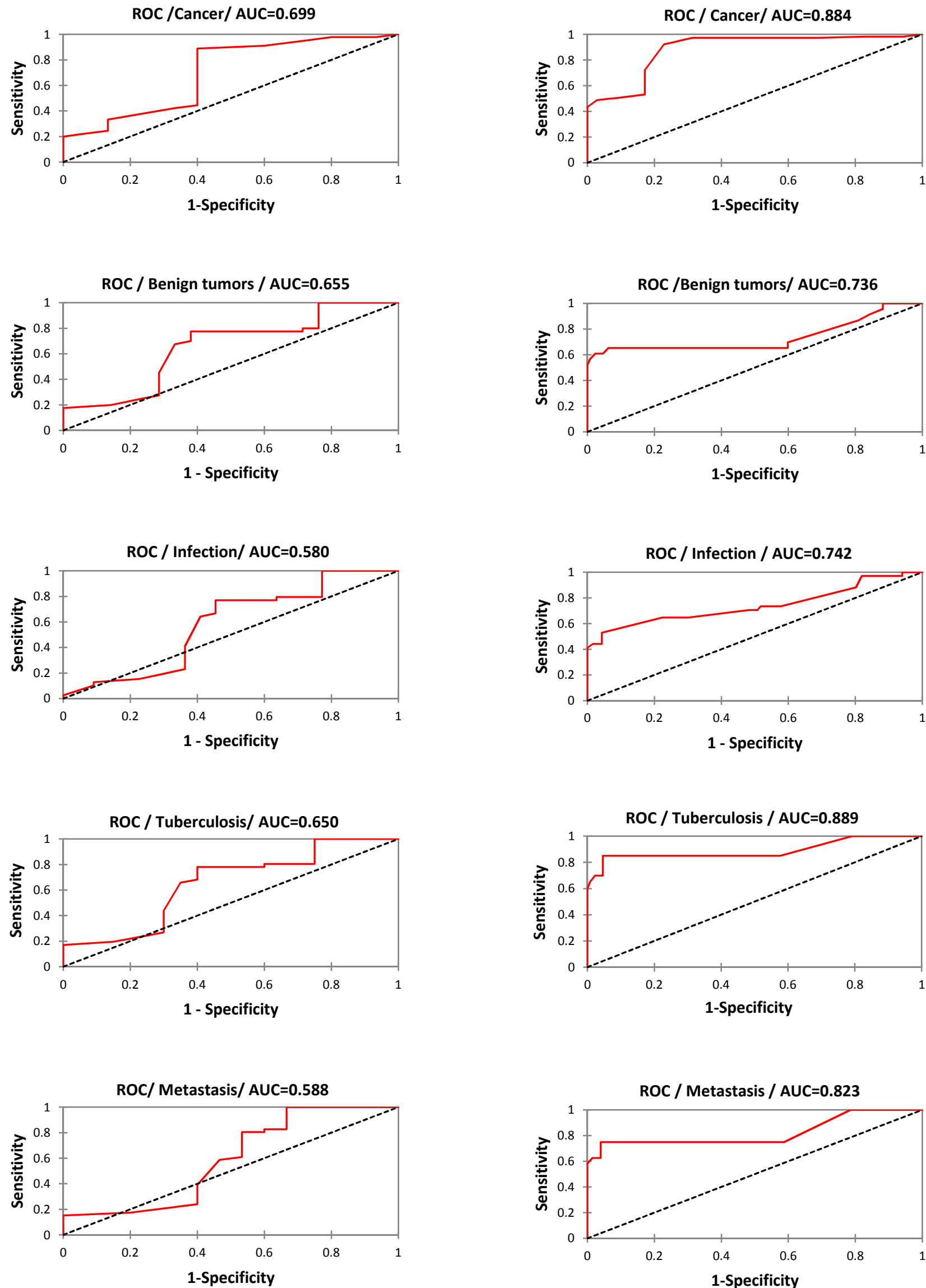

Figure 3. Plots ROC curves for the selected feature sets according to the stepwise forward selection for cancer, benign tumors, infection, tuberculosis and metastasis.

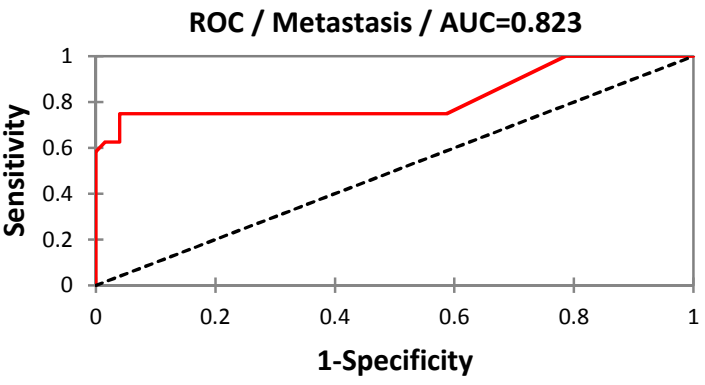

Figure 4. Plots the ROC curves for the selected features sets for cancer, benign tumors, infection, tuberculosis and metastasis according to Principal Components Analysis. 


\section{Conclusion}

In the present work, a novel scheme is proposed for the diagnosis of lung diseases. Features are extracted from detected lesions from lung regions which are segmented automatically. Then, we needed to eliminate redundant variables from the subset extracted because they affect the performance of the classification. We used Stepwise Forward Selection and Principal Components Analysis (PCA). We finally obtained two subsets of features and a comparative study is realized by using the JSRT database. The validation of the proposed method is measured by using the Area Under Curve (AUC) parameter that achieves 0.884 on the cancer database. Compared to the traditional SVM and FCM, The hybrid PCA/FCM/SVM has better accuracy and then Stepwise/FCM/SVM classification. These results prove that the hybridization between PCA, FCM and SVM greatly improve classifier performance. Hence, on the basis of the observed higher efficiency, it can be concluded that the proposed approach is very efficient to classify lung lesions and it may be helpful for radiologists in evaluation of the chest images.

\section{References}

[1] van Ginneken B, Hogeweg L, Prokop M. Computer-aided diagnosis in chest radiography: beyond nodules. Eur J Radiol. 2009;72(2): 226-230.

[2] Bogoni L, Ko JP, Alpert J, et al. Impact of a computer-aided detection (CAD) system integrated into a picture archiving and communication system (PACS) on reader sensitivity and efficiency for the detection of lung nodules in thoracic CT exams. J Digit Imaging. 2012;25(6):771-781.

[3] Lodwick GS. Computer-aided diagnosis in radiology: A research plan. Invest Radiol. 1966;1(1), 72-80.

[4] Campadelli P, Casiraghi E, Valentini G. Lung nodules detection and classification. ICIP205. IEEE International Conference on Image Processing 2005. 2005: I-1117-1120.

[5] Hardie RC, Rogers S, Wilson T, et al. Performance analysis of a new computer aided detection system for identifying lung nodules on chest radiographs. Med Image Anal. 2008;12(3):240-258.

[6] Yeung DS, Ng WWY, Wang D, et al. Localized generalization error model and its application to architecture selection for radial basis function neural network. IEEE Trans Neural Netw. 2007;18(5):1294-1305.

[7] Hamidzadeh J, Monsefi R, Sadoghi Yazdi H. DDC: distance-based decision classifier. Neural Comput Applic. 2012;21(7):16971707.

[8] Al Gindi, A., Rashed, E., \& Sami, M. (2014). Development and Evaluation of a Computer-Aided Diagnostic Algorithm for Lung Nodule Characterization and Classification in Chest Radiographs using Multiscale Wavelet Transform.Journal of American Science, $10(2)$.

[9] Zhou T, Lu H, Zhang J, et al. Pulmonary Nodule Detection Model Based on SVM and CT Image Feature-Level Fusion with Rough Sets. Biomed Res Int. 2016.

[10] Froz BR, de Carvalho Filho AO, Silva AC, et al. Lung nodule classification using artificial crawlers, directional texture and support vector machine. Expert Syst Appl. 2017;69:176-188.

[11] Ben Hassen D, Taleb H, Yaacoub IB, et al. Classification of chest lesions with using fuzzy c-means algorithm and support vector machines. In: International Joint Conference SOCO'13-CISIS'13-ICEUTE'13 (pp. 319-328). Springer International Publishing. 2014.

[12] Ben Hassen D, Taleb H, Ben Yaacoub I, et al. A fuzzy approach to chest radiography segmentation involving spatial relations. IJCA Special Issue on Novel Aspects of Digital imaging Applications (DIA). 2011;(1):40-47.

[13] Ben Hassen D, Taleb, H. Automatic detection of lesions in lung regions that are segmented using spatial relations. Clin Imaging. 2013;37(3):498-503.

[14] van Ginneken B, Stegmann MB, Loog M. Segmentation of anatomical structures in chest radiographs using supervised methods: a comparative study on a public database. Med Image Anal. 2006;10(1):19-40.

[15] Porebski A. Sélection d'attributs de texture couleur pour la classification d'images. Application à l'identification de défauts sur les décors verriers imprimés par sérigraphie [Doctoral dissertation]. Université Lille; 2009.

[16] Jain AK, Duin RPW, Mao J. Statistical pattern recognition: A review. IEEE Trans Pattern Analysis and Machine Intelligence. 2000;22(1):4-37.

[17] Kong H, Wang L, Teoh EK, et al. Generalized 2D principal component analysis for face image representation and recognition. Neural Networks. 2005;18(5):585-594.

[18] Metz CE. ROC methodology in radiologic imaging. Invest Radiol. 1986;21(9):720-733. 